\title{
Model based Control of a Continuous Yeast Fermentation
}

\author{
Andersen, Maria Yolanda; Brabrand, Henrik; Jørgensen, Sten Bay
}

Published in:

American Control Conference

Publication date:

1991

Document Version

Publisher's PDF, also known as Version of record

Link back to DTU Orbit

Citation $(A P A)$ :

Andersen, M. Y., Brabrand, H., \& Jørgensen, S. B. (1991). Model based Control of a Continuous Yeast Fermentation. In American Control Conference (pp. 1329-1334). IEEE.

\section{General rights}

Copyright and moral rights for the publications made accessible in the public portal are retained by the authors and/or other copyright owners and it is a condition of accessing publications that users recognise and abide by the legal requirements associated with these rights.

- Users may download and print one copy of any publication from the public portal for the purpose of private study or research.

- You may not further distribute the material or use it for any profit-making activity or commercial gain

- You may freely distribute the URL identifying the publication in the public portal

If you believe that this document breaches copyright please contact us providing details, and we will remove access to the work immediately and investigate your claim. 


\author{
Maria Yolanda Andersen, Henrik Brabrand, and Sten Bay Jørgensen. \\ Process Design, Dynamics and Control Group \\ Department of Chemical Engineering, Building 229. \\ Technical University of Denmark, DK-2800 Lyngby, Denmark.
}

\begin{abstract}
Control of a continuous fermentation with Saccharomyces cerevisiae is performed by manipulation of the feed flow rate using an ethanol measurement in the exit gas. The process is controlled at the critical dilution rate with a low ethanol concentration of $40-50 \mathrm{mg} / 1$. A standard PI controller is able to maintain the process fainty satisfactory around the critical dilution rate, but better performance is obtained with both an adeptive and a non-adaptive $L O$ controller. However, simulation results indicates that improved performance of the PI controller can be obtained using a simple approximate model to calculate the tuning parameters. The results show that linear, constant gain controllers give satisfactory performance when the process is maintained in a small operating region. Experiments with significant variations in process operation are necessary to demonstrate the advantages of adaptive control.
\end{abstract}

\section{INTRODUCTION}

Control of fermentation processes operated in fed-batch mode is discused in several studies in the literature [1]. In the case of continuous fermentations, the work is concentrated on on-line optimization, which is a most interesting research area due to the possible variability of the microorganisms. The literature reveals that only in a few cases the optimization is combined with an undertying feedback regulator [2], [3]. However, optimization without underiying feedback control is known and demonstrated to be ineffective in performing disturbance rejection [3]. The purpose of this paper is to investigate the continuous fermentation process dynamics and upon this basis to design a feedback controller.

Since fermentation processes are inherently nonlinear the control design must be able to deal efficiently with process variations. There are in principle three different approaches: robust control design, adaptive control, and nonlinear control. In the first case a linear time-invariant model is assumed and the model uncertainty occurring during operation is accounted for in the control design. Such a robust controller can in principle be obtained by designing an ideal controller using a nominal model of the process and then tune the controller on-line for satisfactory performance, or, the entire control design can be done off-line to ensure satisfactory performance for a family of models. The difficulty in this method is, however, that it requires a description of the model uncertainties which reflects only the expected variations in process behaviour. Otherwise, either a too conservative or optimistic control design will result. In the second approach, a linear time-invariant model structure is assumed and parameter variations are tracted during operation. Finally, the third approach is based on knowledge of the non-linear behaviour of the process which is used to derive a non-linear controller.

In this work, the first two mentioned approaches to model based control are investigated since a nonliner model is not available. The robust controller is an internal model controller (IMC) designed by the method given in [4] where a nominal controller is tuned co-line. The adaptive controller is a Linear Quadratic controller combined with on-line identification of an ARMAX-model. Finally, a PI controlker is used. Both simulation and experimental results are presented.
The control problem

In this work, the glucose-limited continuous fermentation with Saccharomyces cerevisiae is investigated. This yeast has a characteristic regulatory behaviour which depends on the substrate concentration. This internal cell regulation is seen by ethanol production if the glucose concentration becomes too high. The produced ethanol is consumed by the cells if the glucose concentration becomes low. This regulatory mechanism within the cells reacts very fast and ethanol can appear in the broth within a minute. Steady state concentrations of biomass, ethanol, and glucose as a function of dilution rate for fixed inlet glucose concentration have the schematic forms shown in fig.1. The dilution rate is defined as the feed flow rate divided by the liquid volume. Ethanol production starts around a critical dilution rate $D_{\text {crit }}$.

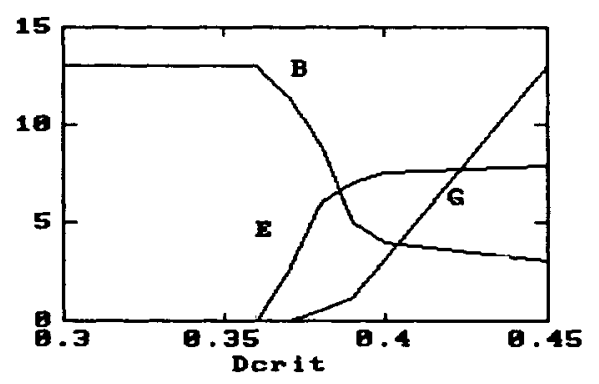

Fig.1: Schematic plot of steady state data as a function of dilution rate for a fixed feed glucose concentration. $y$-axis: concentration of B:biomass, E:ethanol, G:glucose.

The control problem is to maintain the process close to the critical dilution rate in order to obtain maximum productivity of biomass. The critical dilution rate varies for different strains and operating conditions and even within an experiment. Therefore, feedback control is necessary when operation around the critical dilution rate is desired. Otherwise, the process must be operated at a dilution rate well below the critical - and optimal - dilution rate if significant ethanol production is to be avoided.

\section{EXPERIMENTAL SYSTEM}

\section{Fermentor system}

The experimental work was performed using a 201 Chemap fermentor in the pilot plant of Nowo Nordisk A/S. The reactor is equipped with standard measurements of $\mathrm{pH}$, temperature, and dissolved oxygen tension. Oxygen and carbon dioxide concentrations in the exit gas are measured by a mass spectrometer. In addition to these standard measurements the fermentor is equipped with an ethanol sensor and a fluorescence sensor to measure culture NADH. The ethanol sensor is placed in the exit gas behind a teflon membrane chose to the top of the fermentor. The sensor is sensitive to reducing gases and therefore not specific for ethanol. However, in this application only minor amounts of other species which can bias the ethanol measurement, such as acetaldehyde, are expected to be present. The sensor signal is arbitrarily converted to $0-100$ and the output is adjusted to give maximum signal for a liquid concentration 
of $0.5 \mathrm{~g} / \mathrm{l}$. The fluorescence sensor is placed in a fitting with a quarts window through which the liquid fluorescence is measured. This sensor output is also converted to 0-100.

Compater control system

The fermentor is normally controlled by the company's process computer (a FOXBORO 300) and for practical reasons pH and temperature control are performed by this computer. This computer also provides data from the mass spectrometer. The control task is in principle divided in a low and high kevel task each undertaken by a computer. Only the low level computer is interfaced to the process and it receives all the measurements either directly to the I/O board or via a serial communication link from the FOXBORO. This computer also undertakes the low kevel control tasts such as PI control of dissolved orygen and substrate feeding. The low level computer communicates with the high level computer which undertakes more advanced control.

The MODAC (MOdular Data Acquirition and Control) software package runs on the high level computer and the IX (Instrumentation eXpert) software pectage runs on the low level computer. Both are multitasking, real-time, command besed software systems - running on personal computers - developed in house.

Cultivation procedare

The microorganism used is Sacharomyces cerevisiae provided by Novo Nordisk (ATCC 26 785). A standard synthetic medium with gtucose as energy and carbon source and ammonium sulphate as nitrogen source is used. The experiments are started as batch fermentations and run at $30^{\circ} \mathrm{C}, \mathrm{pH} 45,101 \mathrm{air} / \mathrm{min}$, and 0.5 bar pressure. Dissolved aryen tension is controlled at $40 \%$ saturation with a PI controller using the apitator speed as manipulated variable. The liquid worting volume is 81 .

The feed flow and feed glucose concentration can be varied independently by means of two substrate feeds with different glucose concentrations. The two substrate flows are measured by balances and controlled by PI controllers by manipulating the pump speeds. Flow set points for cach substrate flow to obtain an overall feed flow rate and feed glucose concentration are calculated automatically. The feed gluoose concentration is kept constant at $10 \mathrm{~g} / 1$ during the experiments exept when disturbances are imposed.

Details of the experimental system are given in [5].

\section{PROCESS IDENTIFICATION}

In this wort the approach to model based control is to use linear input-output modets for controller design. Adaptive properties of a controlker based on a linear model are essential if operating conditions change significantly as during start-up of a continuous fermentation or during on-line optimization.

The approach to process identification is to perturb the input variables with a Pseudo Random Binary Sequence (PRBS). Proper filtering of the input-output data is done to aroid aliasing and to remove trends.

Perterbation experiment

A characteristic feature of this process is the large change in ethanol concentration within a narrow range of dilution rates around the critical value. Even if a dilution rate is found to give a low ethanol concentration, it is not possible to maintain the low ethand production with the fixed dilution rate during longer time periods. Therefore, it is necessary to perform the experiments in closed loop.

A PI controller is used to control the ethanol signal using the feed flow rate as manipulated variable. Perturbations are imposed on the reference signal to the controller. Tight set point tracting is not necessary in this case as the purpose is to maintain a low ethanol concentration and at the same time allow variations. Fig.2 shows a block diagram of the closed-toop perturbation set-up.

A perturbation experiment is performed with the ethanol reference signal at 10 which corresponds to a liquid concentration of 40-50 $\mathrm{mg} / \mathrm{l}$. A PRBS sequence with a basic period of $6 \mathrm{~min}$ but sampled every $\min$ is generated off-line. The sampling time for the PI controller is $1 \mathrm{~min}$. The feed flow rate is constrained within the limits 3.28 and $2.4 \mathrm{l} / \mathrm{h}$ corresponding to the dilution rates 0.41 and 0.30 $h r^{1}$. The input-output data are shown in fig. 3 .

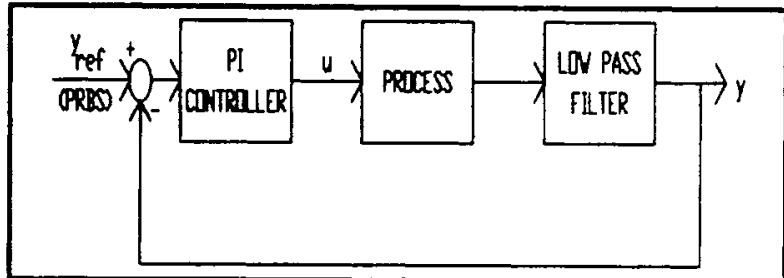

Fig.2: Block diagram of cloced-bop perturbation set-up. u:feed flow rate, $y$ : ethanol measurement.
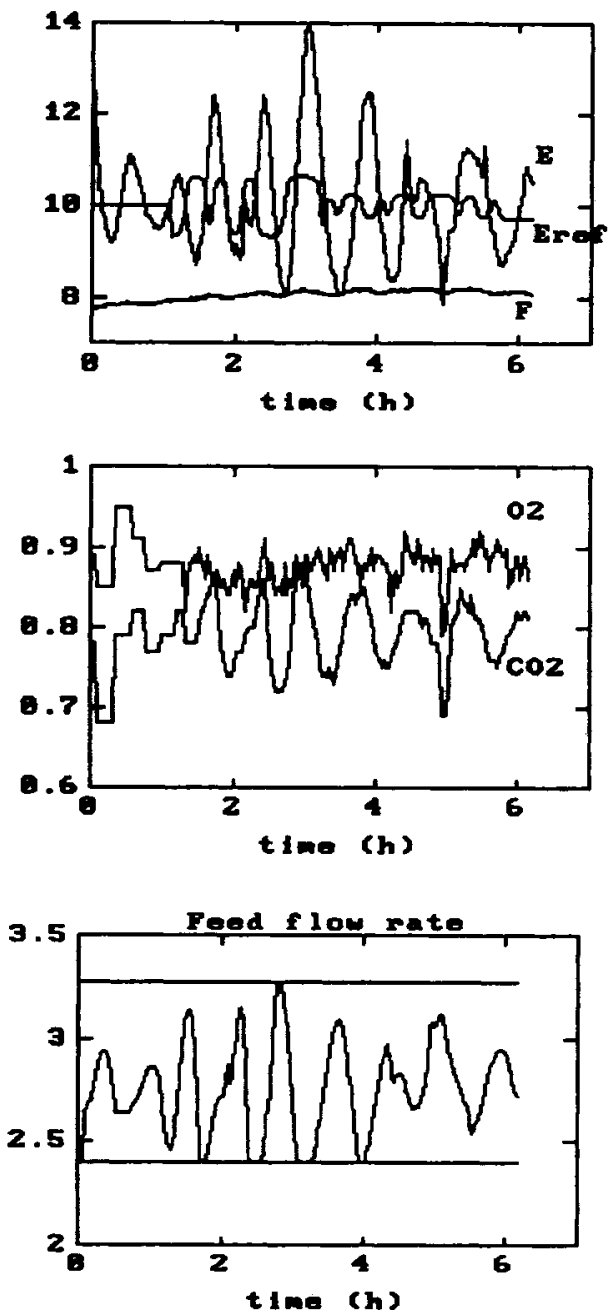

Fig. Perturbation experiment. The $\mathrm{\Delta O}_{2}$ and $\mathrm{ACO}_{2}$ measurements are given as the difference between inlet and outlet concentrations in percentage of air, i.e. $\%(v / v)$. E: ethanol signal (0-100), Eref: ethanol reference in PI controller, F: fluorescence signals $(0-100)$. The feed flow rate is in $1 / \mathrm{h}$. Upper and lower limits for the feed flow rate are indicated by horizontal lines.

The off-gas analysis sample time is increased to $2 \mathrm{~min}$ after the perturbations are started. The ethanol and fluorescence data are filtered with a digital 2 order low-pass filter sampled every $5 \mathrm{sec}$. The filtered signals are then sampled every min. This resampling of measurements from a faster sampled filter avoids aliasing. The corner-frequency for the low-pass filter is $2.3 \mathrm{rad} / \mathrm{min}$ which is slightly below the Nyquist frequency for the $1 \mathrm{~min}$ sampling time. 
Until $t=1 \mathrm{~h}$ the reference to the PI controller is constant at 10 and the ethanol osciliates around the set point. Perturbations are started around $t=1 \mathrm{~h}$ and continued during the following $5 \mathrm{~h}$. The perturbation amplitude is 0.5 during the first $2 \mathrm{~h}$ but it is decreased to 0.2 in order to reduce the variations in the feed flow rate.

Notice the lact of response in the fluorescence signal and in the

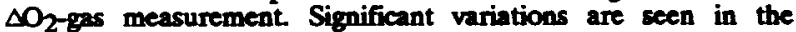
$\triangle \mathrm{CO}_{2}$-gas measurement and the ethanol signal but the first mentioned is generally more noisy that the latter. In addition, the discrete nature of off-ges analy is limits the analysis sample time to $2 \mathrm{~min}$. The same conclusions apply to a RQ measurement calculated as $\triangle \mathrm{CO}_{2} / \mathrm{OO}_{2}$, but this measurement will generally be more noisy than the $\Delta \mathrm{CO}_{2-\text { eas }}$ mescurement. A Flow Injection Analyser for measuring glucose in the broth has been developed. However, this measurement was discarded for control purpose because significant ethand production can results even at low giveose concentrations as seen from fig.1. These considerations regarding which measurements are suitable for identification and control around the particular operating point are supported by glucose pube experiments. Therefore, only the ethanol measurement was used for control. At lower dilution rates the fluorescence signal could also have been chosen as it responds 1-2 min before the ethanol signal and is very sensitive to excessive feeding. If the ethanol measurement is not available, the $\mathrm{ACO}_{2}$-measurement could be used instead.

Or-line identficotion

The off-line calculations are done using MATLAB with the system identification toolbor. The ethanol measurement is chosen as process output and the feed flow rate as the process input. The length of the data set is 281 samples. The mean values of 10.18 for ethanol and $2.751 / \mathrm{h}\left(-D=0.34 \mathrm{hr}^{-1}\right)$ for the feed flow rate are subtracted. No trends are seen in the data and therefore high pess filtering is not done.

Fully parametrized ARX-models of increasing order $n$ are identified to determine the model onder. No further decrease in the residual is obtained and no significant changes are seen in the Bode plots from $n=3$ and above. The input data are not low-pass filtered, hence the transifer function for the lom-pass filter is estimated as well as the process transfer function. The filter transfer function is not recognized neither for a first or second order model. Therefore a third order, fully parametrized model is chosen although the parameters of the A-polynomial were correlated. Fig4 shows the ethanol measurement and the residuals for the off-line identified third order ARX-model.

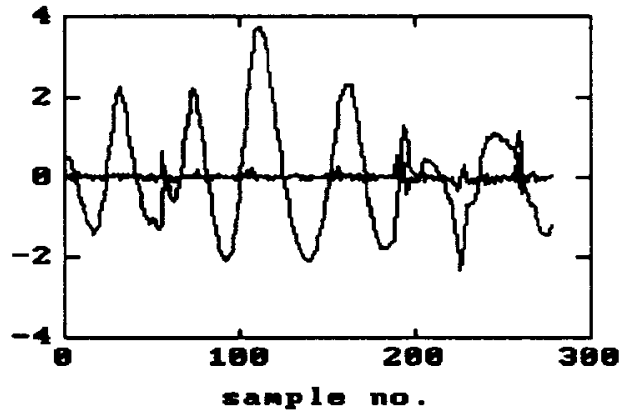

Fig.4: Plot of process output (ethanol measurement) and residuals for off-line identifed third onder ARX-model.

$99.37 \%$ of the variation in the output is explained by the model. The estimated transfer function has the following zeros and poles:

zeros: $1.6563,-0.9088$

poles: $0.9782,0.3562 \pm 0.4698$

The zero outside the unit circle gives an inverse response which can be explained as follows. When the feed flow rate is increased, the ethanol concentration will initially decrease due to the larger flow out of the reactor. But a larger feed flow rate makes the cells produce more ethanol and therefore the ethanol concentration will increase. Fig. 5 shows a simulated step response with the identified transfer function.
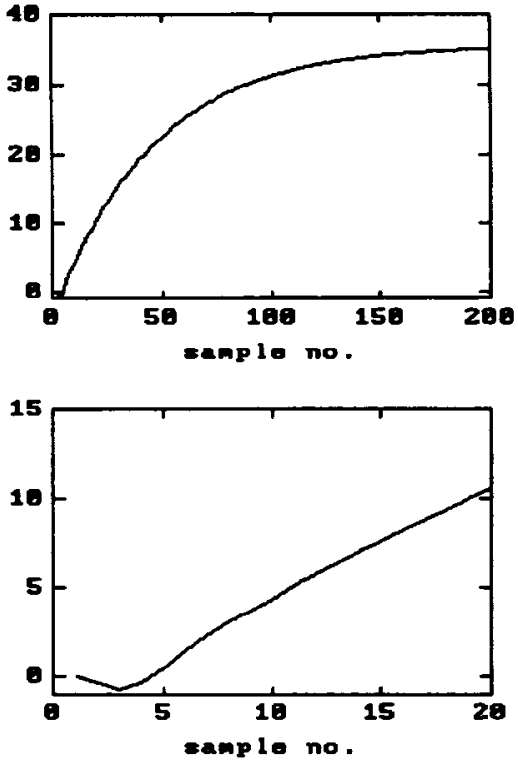

Fig.5: Simulated step response of ethanol signal with identified transfer function model. Lower plot is an expanded plot of the initial response.

The large pole of 0.9782 results in the slow response. It also indicates that the process is ctoee to the stability limit which is consistent with the earlier described observation.

\section{EXPERIMENTAL RESULTS}

An adaptive Linear Quadratic controller is used to control the ethanol concentration by manipulating the feed flow rate. The LQ objective function is

$$
\begin{aligned}
J_{N} & =y^{T}(t+N) Q_{y} y(t+N) \\
& +\sum_{i=1}^{M-1}\left(y^{T}(t+i) Q_{y} y(t+i)+u^{T}(t+i) Q_{x} u(t+i)\right)
\end{aligned}
$$

where the weight martices $Q_{y}$ and $Q_{u}$ are diagonal positive definite matrices. The controller is combined with an on-line identification of an ARMAX-model using the Recursive Extended Least Squares method with a variable forgetting factor. The model is reformulated to a state space representation in the controller and the feedback gains are calculated by iterative solution of the Ricatti equation. Adaptation of the model parameters can be switched off in which case a constant gain LQ controller is obtained. The controller is implemented in MODAC and described in more detail in [6]. Fig.6 shows a block diagram of the adaptive $L Q$ controller.

Before control is started a fully parametrized third order ARMAX model is estimated on-line during the closed-loop experiment previously described. A first order high-pass filter with comer-frequency $0.02 \mathrm{rad} / \mathrm{min}$ is used for both input and output data. The output data are low-pass filtered as previously described.

The transfer function of the estimated model converges after approximately 140 samples (the duration of the PRBS experiment is approximately 300 samples). The scaling factor of the measurement is then changed from 2 to 1 which naturally results in a sharp change in the estimated parameters and thereby in the transfer function. This is seen as an increased gain at high frequencies which remains until the end of the PRBS experiment. The Bode plots of the off-line estimated transfer function and the on-line estimated transfer function at the end of the PRBS experiment are shown in fig.7. 

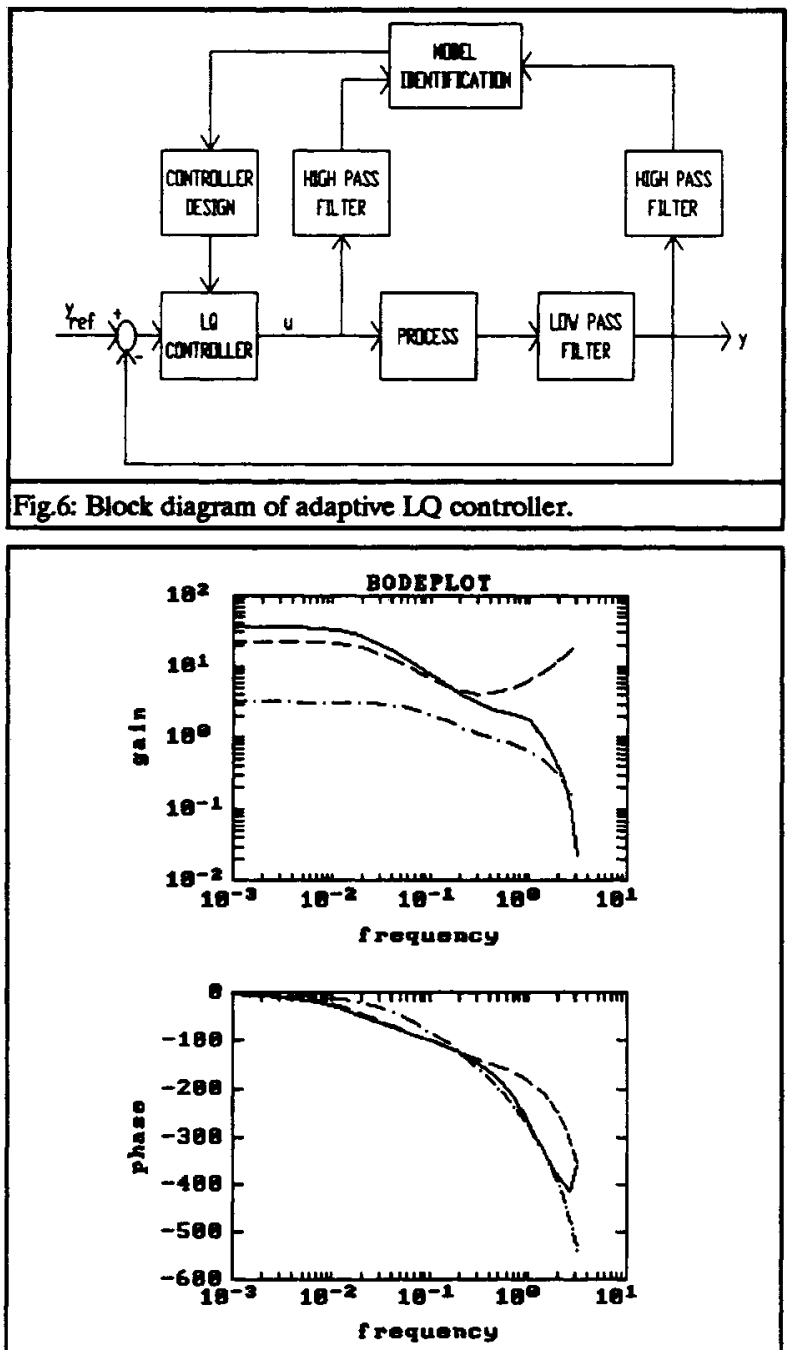

Fig.7: Bode plots of identified transfer functions. Solid line: off-line, dashed line: on-line at $t=6 \mathrm{~h}$, dashed-dotted: on-line at $t=46 h$.

Fig.8 shows the control results with the adaptive $L Q$ controller. The feed flow rate is constrained within the limits 3.28 and $2.41 / \mathrm{h}$ corresponding to the dilution rates 0.41 and $0.30 \mathrm{hr}^{-1}$. The control weights in the $L Q$ objective function are 5 and 1 respectively for the input and output variables.

At $\mathrm{t}=6 \mathrm{~h}$ the perturbation - and the PI controller - is stopped and the LQ controller is used to control the ethanol concentration at the desired value of 10. Convergence of the Ricatti equation solution is ensured by 20 iterations in each of 4 samples before the controller is started. Thereafter, 2 iterations in each sample is used. During the first 2 hours approximately, the control performance is fairty bad as seen in the variations in the ethanol measurement. Large variations are also seen in the feed flow rate. The reason for this is the mismatch between the process transfer function - which can be represented by the off-line estimated transfer function - and the on-line estimated transfer function, i.e. the model in the controller. But after approximately 2 hours (120 samples) the model has converged and the false high gain in the high frequency range is no longer present.
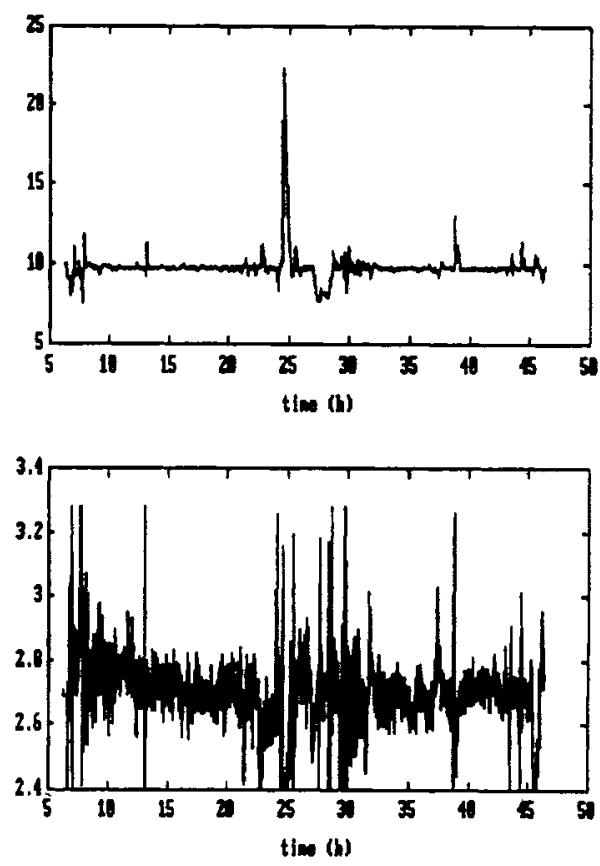

Fig.8: Control with adaptive LQ controller. Upper plot: controlled variable, lower plot: manipulated variable. $Q U=5, Q Y=1$.

After the initial adaptation follows a long period of nearty 15 hours in which the ethanol concentration is kept nicely at the desired set point of 10 with only minor disturbances. At $t=24 \mathrm{~h}$ a large load disturbance is imposed consisting in an increased feed glucose concentration from 10 to $20 \mathrm{~g} / 1$ during 2 sample intervals. The controller decreases the feed flow rate to the lower limit. The ethanol measurement is back at the set point after $\mathbf{4 0} \mathrm{min}$ but shoetly after a disturbance follows having the same form and magnitude as the the one seen approximately $1.5 \mathrm{~h}$ before the imposed load disturbance. During a short period of 1.4 hours the ethanol set point is decreased from 10 to 8 (from $t=26.9-28.3 \mathrm{~h}$ ). This set point change is very small as it corresponds to a $20-30 \mathrm{mg} / 1$ decrease in liquid ethanol concentration. The response following the set point increase is quite oscillatory.

Control with the non-adaptive LQ controller is shown in fig.9. The same input and output weights and limits on the feed flow rate as for the adaptive $L Q$ are used. Estimation is set off at $t=46 \mathrm{~h}$ and $a$ Bode plot of the transfer function estimate at this time is shown in fig.7. This model has a significantly lower gain than the other two shown.

The performance of the non-adaptive LQ controller is similar but clearly better than the adaptive $L Q$. Less variations and disturbances are seen with the non-adaptive $L Q$. The effect of the load disturbance is eliminated within $30 \mathrm{~min}$. These results are, however, not sufficient to conclude that adaptation should be avoided. In order to demonstrate the advantages of adaptive control larger set point changes should be performed. A step change in the feed glucose concentration would also result in a significant change in process gain as the biomass concentration would change. In addition, it is believed that improved performance of the adaptive controller can be obtained by better adjustment of the parameter adaptivity to obtain a less alert controller. A feasible operation form would be to use the LQ controller without adaptation and only swritch estimation on if the controller performance becomes unsatisfactory due to changed process dynamics. During adaptation addition of PRBS signals to the inputs will ensure sufficient conditions for identification. 

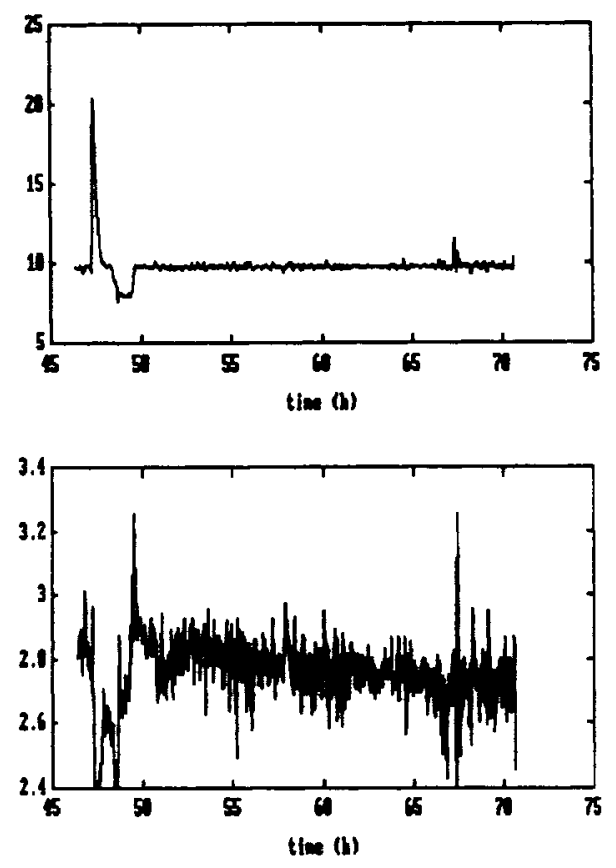

Fig.9: Control with non-adaptive LQ controller. Upper plot: controlled variable, lower plot: manipulated variable. $Q U=5$, $\mathrm{QY}=1$.

The third controller used experimentally is the standard PI controller used for the closed-toop perturbation experiment previously described. From fig. 3 it is seen that the set point tracking performance is bad. No systematic tuning procedure was used and tuning of the PI controller was therefore time consuming. The performance of this controller for disturbance rejection is seen in fig.10. Two disturbances are imposed as the first one was not quite as large - in terms of extra amount of added glucose - as the ones imposed in the case of $L Q$ control.

Large oscillations are seen in the ethanol measurement before the first disturbance. The ethanol measurement is back around the set point - but still oscillating - after one hour. The maximum value for the ethanol measurement is somewhat larger for the second disturbance. In this case it also takes 1 hour to return to the set point but the following oscillations are clearly reduced. This controller also reduces the feed flow rate to the lower limit after the disturbances.

\section{SIMULATION RESULTS}

It is interesting to investigate the performance of an IMC controller which is a simple model based controller. The off-line identified transfer function model is used for control design and simulation of the process.

A two step design method for the IMC controller is used as described in [4]. The design is done in discrete time. The first step is to factorize the model into a stable part and a part containing the zero outside the unit circle. The IMC controller is then obtained by inverting the stable part of the model and multiplying with a low-pass filter. The zero close to $(-1,0)$ of the model transfer function becomes a pole in the control transfer function which can cause intersample rippling ([4]). Therefore, this pole is cancelled and placed at the origin. The time constant of the filter is the only tuning parameter which is adjusted on-line to obtain satisfactory performance and robustness. A first order filter is used and the tuning parameter $\alpha$ is in the range 0 to 1 . A value of 0 leads to a controller which is the inverse model and 1 leads to open-loop control.
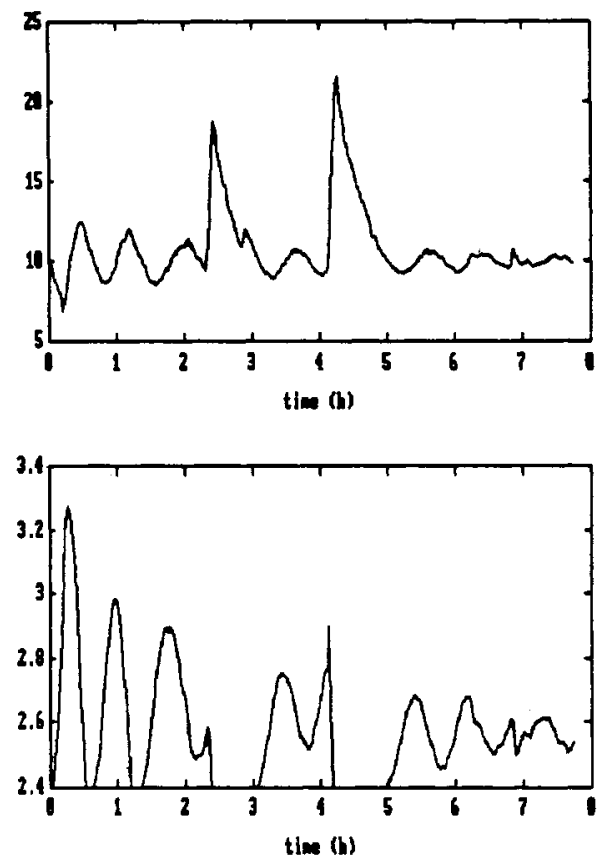

Fig.10: Performance of PI controller for disturbance rejection. Two load disturbances are imposed. Upper plot: controlled variable, lower plot: manipulated variable. $K_{c}=0.08, \tau=2.7 \mathrm{~min}$ until $t=1.3$ $\mathrm{h}$, then increased to $4 \mathrm{~min}$.

The performance of the IMC controller for disturbance rejection and set point tracking is compared to the non-adaptive $L Q$ and a $P I$ controller. The disturbance is simulated as a load disturbance in the ethanol measurement. The parameters of the off-line identified model are used in the LQ controller. The PI controller is tuned using the method described in [7] in which IMC design is used to calculate the PI tuning parameters. The step response shown in fig.5 shows that the process has a large time constant versus deadtime. Therefore, the model is approximated to an integrator phus deadtime in order to avoid a slow load response [7]. Having the process gain and deadtime from the step response (these are 0.6667 and $5 \mathrm{~min}$ respectively) the two PI tuning parameters are calculated by specifying the time constant of the closed-loop response. This response time constant need to be larger than the deadtime in onder to avoid oscillatory response. A closed-loop time constant of $10 \mathrm{~min}$ is used. The IMC tuning parameter has an optimal value around 0.8 as less good performance results when it is increased or decreased. Fig.11 shows the performance of the three controllers.

Similar performance is obtained for all three controllers. The most significant difference is seen in the disturbance rejection where the IMC and PI controllers both have a more sluggish response than the LQ controller. Using the same PI tuning parameters as for the experimental results gives a much more oscillatory response. Although the PI controller as such does not have any information of the process dynamics in form of a model, this model based tuning seems to be an efficient method to improve the PI controller performance.

\section{CONCLUSIONS}

A perturbation experiment is successfully performed around the critical dilution rate. Closed-loop operation is necessary at this operating point in order to maintain a low ethanol concentration. For this particular set-up the ethanol measurement is found to be the most suitable measurement for control. An off-line identified model, using the feed flow rate and the ethanol measurement, reveals inverse response for this pair of input output variables. 

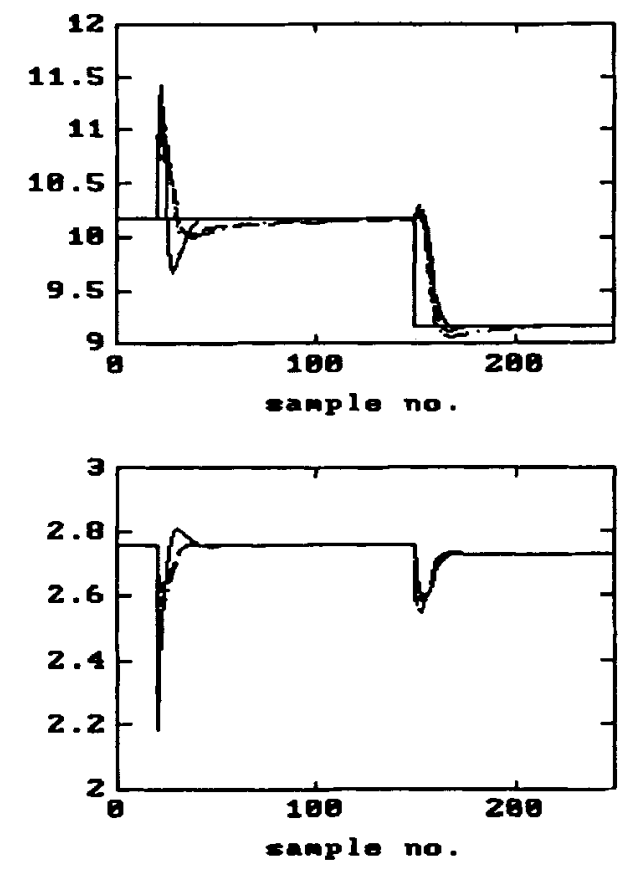

Fig 11: Simulated LQ (solid), MMC (dashed), and PI (dashed dottet) control. Upper plot: controlled variable, lower plot: manipulated variable. IMC: $\propto=0.80,1 \mathrm{Q}: Q U=5, Q Y=1, P I: K_{c}=0.1667, \tau_{F}=25$.

Tight control is obtained during almost $70 \mathrm{~h}$ at the critical dilution rate using a $L Q$ controller both with and without adaptation. The non-adaptive $L Q$ has better performance than the adaptive $L O$ controller. It is necessary to test the adaptive and non-adaptive controlkers for changes in process conditions leading to significant changes in process gain in order to reveal the expected advantages of adaptive control.
It is possible to maintain the process at the desired operating point also with a standard PI controller although the obtained control is not as tight as with the LO controllers. The constraints in feed flow rate confines the control action when the large load disturbance is imposed, but still the time needed to eliminate the effect of the disturbance is twice as long for the PI controller compared to the non-adaptive $\mathbf{L Q}$.

Simulations show that similar performance is obtained with an IMC, LQ, and PI controller all designed on the basis of the experimentally identified model. This indicates that improved performance can be obtained experimentally using model based tuning of the PI controller. The LQ controller is tuned for faster response than the IMC and PI controllers. Such tuning for fast response seems feasible for adaptive control. The IMC and PI controllers will probably have to be detuned for practical application where changes in fermentation dynamics can occur. For this detuning a priori knowledge of the model uncertainty is required.

\section{REFERENCES}

(1] Jørgensen,S.B. \& Jensen,N., (1989). Dynamics and control of chemical reactors - Selectivety sinved, DYCORD'89, Maastricht, Holland.

[2] Shi,Z,Shimizu,K,Iijima,S.,Morisue,T. \& Kobayashi,T., (1990). Adaptive on-line optimizing control of a lactid acid fermentation, J. Ferm. \& Bioeng, vol.70, pp.415-419.

[3] Andersen,M.Y.,Asferg,L,Brabrand,H,,Karim,N. \& Jorgensen,S.B., (1989). On-line optimizing control of a simulated continuous yeast fermentation, proc., ACC 1989, Pittsburg.

[4] Morari,M. \& Zafiriou,E,(1989). Robust process control, Prentice-Hall.

[5] Andersen,M.Y, (1990). Multivariable identification of a con. tiruous yeass fermentation. Ph.D. thesis, Department of Chemical Engineering. Technical University of Denmark, Lyngby.

[6] Brabrand,H.Jensen,N. \& Jorgensen,S.B., (1990). MIMOSC a tool for real-time multivariable identification and adaptive control of chemical processes. Nondic CACE symposium, November 15-16, Lyngby, Denmark.

[7] Chien,I.-L. \& Fruehauf,P.S, (1990). Consider IMC auning to improve controller performance. Chemical Engineering Progress, October, pp.33-41. 